\title{
Cefaléia Associada a Atividade Sexual com Vasoespasmo
}

Kleber Edson Kawagoe*

Deusvenir de Souza Carvalho**

Acary Souza Bulle Oliveira***

\section{RESUMO}

A cefaléia associada a atividade sexual (CAAS) é uma entidade dividida em trés tipos: contração muscular, vascular e de baixa pressão liquórica. O tipo vascular é comumente confundido com hemorragia subaracnóidea e, portanto, está indicada a investigaçăo com exames complementares. O achado de vasoespasmo cerebral encontrado após ruptura aneuris. mática e em algumas outras situaçðes, mas não tem sido descrito em casos de CAAS. Descrevemos um caso de CAAS em uma paciente na qual o estudo angiogrático demonstrou vários estreitamentos segmentares, reversiveis, em diversos territórios arteriais cerebrais.

\section{UNITERMOS}

Cefaléia, atividade sexual, vasoespasmo, hemorragia subaracróidea.
* Residente de Neurologia da Escola Paulista de Medicina - UNIFESP.

** Professor-Adjunto e Chefe do Setor de Investigação e Tratamento das Cefáléias da Disciplina de Neurologia da Escola Paulista de Medicina - UNIFESP.

*** Chefe do Setor de Doenças Neuromusculares da Disciplina de Neurologia da Escola Paulista de Medicina - UNIFESP.

\section{INTRODUÇÃO}

A cefaléia pode ocorrer durante a atividade sexual, associada ao intercurso sexual ou independente dele, por exemplo, masturbação. A relação entre a hemorragia subaracnóidea e o aparecimento de cefaléia durante a atividade sexual, particularmente relacionado ao orgasmo, já é bem conhecida, mas nem sempre a cefaléia que ocorre nesse momento é necessariamente indicativa de grave doença neurológica ${ }^{1,2,3}$.

Em 1970, Kriz foi o primeiro a descrever uma modalidade de cefaléia associada ao coito. Várias denominações foram atribuídas a essa condição, tais como cefaléia orgásmica, cefaléia benigna do coito, cefaléia coital. Essa forma benigna de cefaléia sexual, como a própria terminologia indica, não é associada a causas secundárias. Essa condição já teve várias designações, sendo mais recentemente denominada pela Classificação da Sociedade Internacional de Cefaléia como cefaléia associada a atividade sexual $(\mathrm{CAAS})^{5}$. Estreitamentos vasculares cerebrais reversíveis (vasoespasmo) não são encontrados nesse tipo de cefaléia, acontecendo geralmente após ruptura de aneurismas cerebrais. Relatamos o quadro de uma paciente de 39 anos que apresentou uma cefaléia relacionada ao intercurso sexual en cuja investigação angiográfica foram encontradas anormalidades compatíveis com vasoespasmo em diversos territórios arteriais cerebrais. Esse achado é infrequiente, segundo os resultados de uma revisão da literatura ${ }^{11}$.

\section{RELATO DE CASO}

Paciente do sexo feminino, 38 anos de idade, sem passado de cefaléia, tanto pessoal como familiar, referia que há uma semana, durante relação sexual, sentiu cefaléia latejante, de moderada intensidade, localizada no vértice, que durou 3 dias, sem fatores de melhora ou de piora. Passou bem um dia e em seguida apresentou novo episódio na mesma situação, porém de maior intensidade, durando 3 dias, acompanhada de náuseas e vômitos e com alívio após uso de $500 \mathrm{mg}$ de ácido acetilsalicílico. Negava traumatismos encefálicos, uso de medicamentos, incluindo hormônios, previamente aos episódios de cefaléia. Relatava antecedente de neurocisticercose tratada há 
16 anos. O exame físico geral e neurológico da paciente estavam normais.

A paciente foi internada para investigação. As provas reumatológicas: anticorpo antineutrófilo, anticorpo antinuclear, anticorpo anti-DNA, células LE, fator reumatóide e anticardiolipina foram negativos. A dosagem do complemento mostrou níveis normais. A velocidade de hemossedimentação foi normal. As sorologias para hepatite $B$, hepatite $\mathrm{C}$ e para lues resultaram negativas. $\mathrm{O}$ estudo do líquido cefalorraquiano (LCR) foi normal e a tomografia de crânio (TC) demonstrou calcificação única frontal direita, provavelmente devido à neurocisticercose, relatada pela paciente. A ressonância nuclear magnética encefálica não revelou anormalidades. A angiografia dos quatro vasos cerebrais demonstrou vários estreitamentos vasculares em diversos territórios, compatíveis com vasoespasmo ou com vasculite (Figuras 1 e 2). Estando no aguardo dos exames reumatológicos, e como os resultados da angiografia sugeriam arterite, foi, então, optado por tratamento com corticosteróides. A paciente permaneceu assintomática por dois meses. Uma nova angiografia, nessa época, foi normal, sem vasoespasmos.

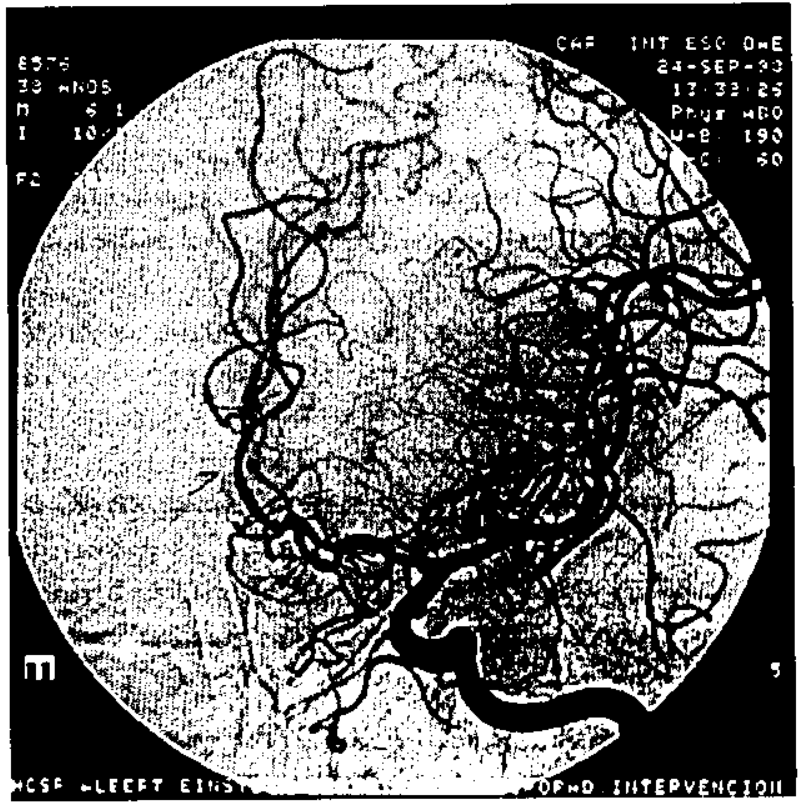

\section{FIGURA 1}

Imagem angiográfica da artéria carótida interna esquerda (OAE), com estreitamentos (setas).

\section{DISCUSSÃO}

A CAAS é dividida em três grupos ${ }^{6,7}$, baseados nos dados clínicos e no provável mecanismo fisiopatológico. $O$ primeiro grupo refere-se a uma cefaléia tipo contração muscular, correspondendo a $24 \%$ das CAAS, que

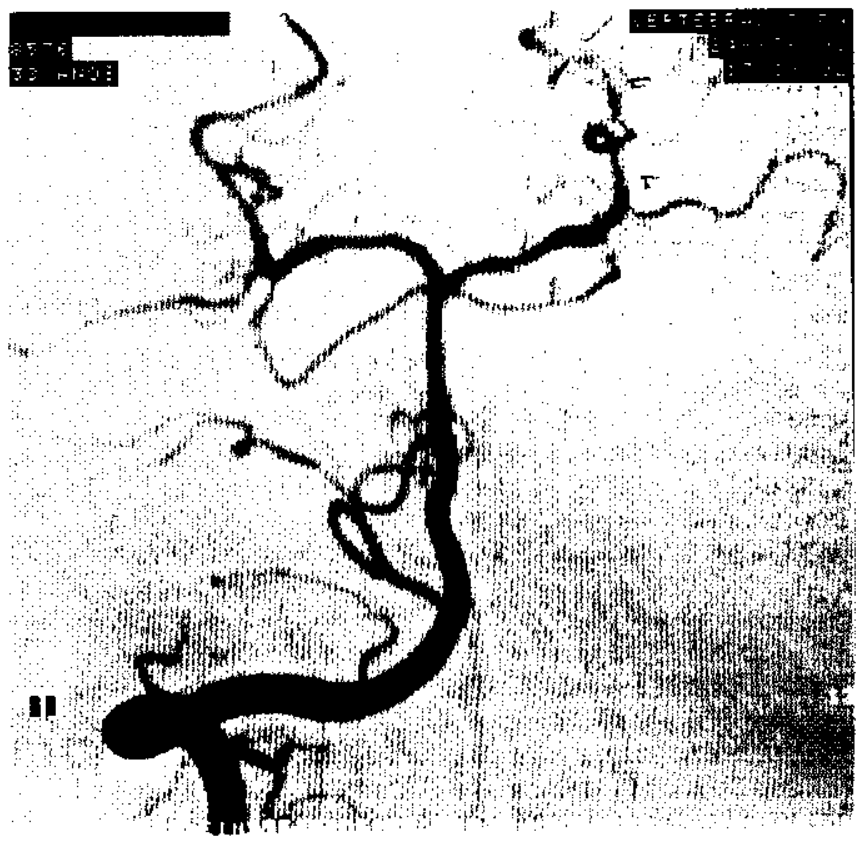

FIGURA 2

Imagem angiográfica da artéria vertebral direita (PA), com estreitamentos vasculares (setas).

geralmente se desenvolve no início do intercurso sexual, de localização occipital ou generalizada, é caracterizada como um peso ou aperto, podendo durar horas ou dias, e é relacionada à contração muscular que ocorre durante o período pré-orgasmo ${ }^{7}$. O tratamento baseia-se em técnicas de relaxamento e orientação postural. $O$ segundo grupo, o mais comum, refere-se a uma cefaléia vascular que corresponde a $69 \%$ dos casos, ocorre próximo ou durante ao orgasmo, usualmente bioccipital ou bifrontal, descrita como explosiva e pulsátil, dura minutos, horas, até dias. Sua fisiopatologia ainda é obscura, podendo estar relacionada a mudanças autonômicas e vasculares que ocorrem nesse momento? ${ }^{7}$, sendo, portanto, um subtipo da cefaléia associada ao esforço físico. Porém, essa cefaléia pode acontecer mesmo nos pacientes com atitudes passivas, ou, segundo alguns.autores, seria uma variante da enxaqueca ${ }^{8,9}$. Essa hipótese é baseada na semelhança dos sintomas com a enxaqueca, a incidência maior de enxaqueca em pacientes com CAAS e a eficácia dos $\beta$ bloqueadores no seu tratamento. A terapêutica ainda inclui indometacina, baseada principalmente na hipótese de ser um subtipo da cefaléia associada ao esforço físico ${ }^{10}$. $O$ terceiro grupo, o mais raro ( $7 \%$ dos casos), refere-se a uma cefaléia de baixa pressão liquórica, com o aparecimento no momento do orgasmo, dura dias ou semanas, é relacionada à postura ortostática e é provavelmente devida à perda de LCR por fístula peridural, semelhante à dor que ocorre após punção de líquido lombar. Seu tratamento 
consiste em repouso prolongado ${ }^{6}$ e demais orientações para o tratamento de cefaléia pós-punção.

A CAAS é quatro vezes mais freqüente no sexo masculino que no sexo feminino, mais comum entre a terceira e a sexta década de vida ${ }^{8}$. Os fatores potencialmente predisponentes incluem: hipertensão arterial, obesidade, condição física pobre e estresse psicossocial.

Os sinais e sintomas que indicam patologias intracranianas sérias incluem: um primeiro episódio de CAAS do tipo vascular, vômitos, alteração do nível de consciência, meningismo, dor persistente por mais que 24 horas e déficits neurológicos focais ${ }^{8}$. A presença de algum desses fatores indica a necessidade de internação e investigação com exames complementares: LCR, TC e angiografia.

O principal diagnóstico diferencial deve ser feito com hemorragia subaracnóidea após ruptura aneurismática ou por complicação de malformações arteriovenosas. $\mathrm{O}$ rompimento de aneurismas durante o intercurso sexual resultando em hemorragia subaracnóidea varia entre $3,8 \%$ e $12 \%$, e em $4,1 \%$ das malformações arteriovenosas 2.3 .

$O$ achado angiográfico de estreitamentos vasculares segmentares de diferentes territórios arteriais descrito nessa paciente pode ser encontrado em muitas outras situações: hemorragia subaracnóidea, uso de drogas simpatomiméticas, manipulação cirúrgica, trauma cranioencefálico, meningite, arterites, arteriosclerose, feocromocitoma e enxaqueca ${ }^{11}$. O próprio procedimento angiográfico pode provocar espasmo arterial em pacientes com enxaqueca, particularmente quando contrastes iônicos são usados, injeções repetidas num mesmo segmento arterial e o uso de cateteres rígidos e de grande calibre. Nesses casos o procedimento é associado a grande desconforto do paciente durante a injeção de contraste ${ }^{11}$. Outra possibilidade que deve ser considerada é uma entidade descrita como "vasoconstrição cerebral segmentar reversível", com achados angiográficos similares, porém apresenta déficits neurológicos transitórios ou permanentes associados a cefaléia intensa ${ }^{12}$. Os dados dessa paciente não a enquadram em nenhuma dessas condições relatadas. Comparando com a literatura, encontramos a descrição de um caso que se assemelha ao dessa paciente, feita por Silbert et al. ${ }^{11}$, e concordamos com esses autores no que se refere à CAAS ser listada entre as condições associadas a vasoconstrição segmentar de vasos cerebrais.

\section{SUMMARY}

Headache Associated with Sexual Activity

Headache associated with sexual activity (HASA) can be divided into three types: a tension type, a vascular or migraine type, and a low cerebrospinal fluid pressure type. The migraine type commonly taken as subarachnoid hemorrhage should have a CT scan, lumbar puncture and cerebral angiogram. Cerebral vasospasm may occur associated to ruptured saccular aneurysm and other causes of intracranial bleeding. A reversible cerebral segmental vasocronstriction in a patient on clinical feature of headache associated to sexual activity is decribed.

\section{KEYWORDS}

Headache, sexual activity, vasospasm, subarachnoid hemorrhage.

\section{Referências}

1. Fisher CM. Headache in cerebrovascular disease. In: Vinken PJ \& Bruyn GW (Eds.). Handbook of Clinical Neurology. New York, North Holland Publishing Co, 1968. p.147-8.

2. Lundberg PO \& Osterman PO. The benign and malignant forms of orgasmic cephalgia. Headache, 14:164-65, 1974.

3. Locksley HB. Natural history of subarachnoid hemorrhage, intracranial aneurysms and arteriovenous malformations: Based on 6368 cases in the cooperative study. J Neurosurg. 25:219-39, 1966.

4. Kriz K. Coitus as a factor in the pathogenesis of neurological complications. Cesk Neurol Neurochir, 33:162-67, 1970.

5. Headache Classification Committee of the International Headache Society. Classification and diagnostic criteria for headache disorders, cranial neuralgias and facial pain. Cephalalgia, 8 (supp7):19-25, 1988.

6. Paulson GW \& Klawans HL. Benign orgasmic cephalgia. Headache, 13:181-7, 1974.

7. Lance JW. Headaches related to sexual activity. J Neurol Neurosurg Psychiatry, 39:1226-30, 1976.

8. Johns DR. Benign sexual headache within a family. Arch Neurol, 43:1158-60, 1986.

9. Porter M, Jankovic J. Benign coital cephalalgia. Arch Neurol, 38:710-12, 1981.

10. Braun A, Klawans HL. Headaches associated with exercise and sexual activity. In: Vinken PJ \& Bruyn GW (Eds.). Handbook of Clinical Neurology. New York, Elsevier Science Publishing Co, 1985. p.373-82.

11. Silbert PL, Hankey GJ, Prentice DA et al. Angiographically demonstrated arterial spasm in a case of benign sexual headache and benign exertional headache. Aust NZJ Med, 19:466-8, 1989.

12. Call GK, Fleming MC, Sealfon $S$ et al. Reversible cerebral segmental vasoconstriction. Stroke, 19:1159-70, 1988.

\section{Endereço para correspondência}

Deusvenir de Souza Carvalho

Disciplina de Neurologia

Rua Pedro de Toledo, 650 - Ed. Jairo Ramos - $2^{\circ}$ andar, sala 48 CEP 04039-002

e-mail: deusveni@provida.org.br 